\title{
¿DIVERTÍCULO URETRAL TRAS DESCENSO ANORECTAL ASISTIDO POR LAPAROSCOPIA (DARAL) EN MALFORMACION ANORECTAL: ES SIEMPRE NECESARIO RESECAR EL DIVERTICULO?
}

\author{
Pedro José López, Miguel Guelfand', Lorena Angel, Angélica Paulos, Yair Cadena, José M. \\ Escala, Nelly Letelier y Ricardo Zubieta.
}

Departamento de Urología y Cirugía Pediatrical. Hospital de Niños Dr. Exequiel Gonzalez Cortes. Santiago. Chile.

\begin{abstract}
Resumen.- OBJETIVO: Con el uso cada vez mayor de la cirugía mínimamente invasiva, el divertículo uretral tras la cirugía anorectal se ha convertido en un problema. Los pocos casos descritos se han manejado con extirpación quirúrgica. Nosotros describimos un caso de divertículo uretral tras descenso anorectal asistido por laparoscopía (DARAL) con un resultado exitoso despues de un período de vigilancia activa.
\end{abstract}

MÉTODO: Se trata de un paciente de sexo masculino, nacido a término, portador de una malformación anorectal (MAR) alta con fístula recto-prostática fue sometido a una colostomía el primer día de vida. También portador de malformaciones asociadas; reflujo bilateral de bajo grado, riñón en herradura y una hemi-vértebra

\section{CORRESPONDENCIA}

Pedro José López E.

Departamento de Urología

Hospital Exequiel González Cortes

Barros Luco 3301

San Miguel. Santiago. (Chile)

pejotalopez@yahoo.com

Aceptado para publicar: 7 de octubre 2009 torácica; sin embargo, sin signos de médula anclada. Se inició profilaxis antibiótica.

RESULTADOS: A los 3 meses de edad, fue sometido a un DARAL con un abordaje abdominal de 3 puertos. Despues de la completa disección del intestino distal, la fístula recto-prostática fue identificada y ligada con clips metálicos. Un trocar de $10 \mathrm{~mm}$ fue insertado a través del centro del complejo esfinteriano, que fue previamente identificado bajo visión laparoscópica durante la estimulación eléctrica perineal. El descenso anorectal se llevó a cabo sin tensión. La vejiga permaneció drenada con catéter uretral por 14 días. En el $18^{\circ}$ día post-operatorio, una cistografía miccional mostró una imagen diverticular de $15 \times 5 \mathrm{~mm}$ a nivel de la uretra membranosa. Despues de 6 meses, una nueva cistografía miccional mostró una uretra normal sin signos de divertículo ni estenosis; persistencia de reflujo grado 2 a derecha y resolución de reflujo a izquierda. Al año de vida la colostomía fue cerrada sin problemas. Seis meses después, se ha mantenido libre de infección urinaria y su micción es con flujo normal.

CONCLUSIÓN: Este artículo sugiere que el DARAL es un abordaje factible para MAR, aunque el divertículo uretral es una de las principales preocupaciones. Puede evolucionar sin complicaciones, y eventualmente resolverse en forma espontánea. La vigilancia activa puede ser una opción en pacientes asintomáticos seleccionados, sin embargo se recomienda un mayor seguimiento para constituir una mejor evidencia que apoye esta medida.

Palabras clave: Laparoscopia. Pediátricos. Malformación anorectal. Complicaciones. Uretra. Divertículo. 
Summary.- OBJECTIVES: With the increased use of minimally invasive surgery, the urethral diverticulum after anorectal surgery has become an issue. The few cases reported have been managed by surgical excision. We hereby report a case of urethral diverticulum after a laparoscopically-assisted anorectal pull-through (LAARP) procedure with a successful outcome after a period of active surveillance.

METHODS: A full-term boy who displayed a high anorectal malformation (ARM) and a recto-prostatic fistula underwent colostomy on the first day. He also showed associated malformations: bilateral low-grade reflux, horseshoe kidney and thoracic hemivertebrae; however, there were no signs of spinal cord tethering. Antimicrobial prophylaxis was started.

RESULTS: At the age of 3 months, he underwent a LAARP with a 3 abdominal-port approach. After complete dissection of the distal bowel, the recto-prostatic fistula was identified and tied with metallic clips. A $10 \mathrm{~mm}$ trocar was inserted through the centre of the sphincteric complex, which had been previously identified under laparoscopic view during perineal electrical stimulation. The anorectal pull-through was accomplished without tension. The bladder remained stented for 14 days. On the 18th postoperative day, a voiding cystourethrogram (VCUG) showed a $15 \times 5 \mathrm{~mm}$ image of the diverticulum at the level of the membranous urethra. After 6 months, a new VCUG showed a normal urethra with neither signs of the diverticulum nor strictures; persistence of grade 2 reflux on the right side and resolution of the reflux on the left. When the boy was one year old his colostomy was closed uneventfully. Six months later he had not come into the emergency since the operation and voided with a normal flow.

CONCLUSION: This report suggests that LAARP is a feasible approach for ARM, although urethral diverticulum is a major concern. It may evolve without complications, and eventually resolve spontaneously. Active surveillance might be an option in selected asymptomatic patients; however a longer follow-up is advised to constitute better evidence supporting that policy.

Keywords: Laparoscopy. Paediatrics. Anorectal malformations. Complications. Urethra. Diverticulum.

\section{INTRODUCCIÓN}

La reparación de una malformación anorrectal es un procedimiento quirúrgico complejo y puede asociarse a complicaciones como daño uretral, especialmente en niños con fístula recto-uretral $(1,2)$. El riesgo de complicaciones es significativo debido a la proximidad física entre la uretra y la bolsa rectal y también debido a la anatomía anormal presentada por la fístula recto-uretral (3). El conocimiento de la anatomía pre operatoria y la experiencia quirúrgica es fundamental para evitar lesiones innecesarias (4).

Después de el reporte de Peña y Hong con pacientes con malformación anorrectal (MAR) reparada a través de anorectoplastia sagital posterior (ARPSP) que desarrolló continencia total en el $25 \%$ de los casos (5), Georgeson et al crearon el descenso anorrectal asistido por laparoscopía tratando de prevenir grandes incisiones que podrían dañar el esfínter muscular y el suministro nervioso (6). Actualmente, el descenso anorrectal asistido por laparoscopía (DARAL) ha sido propuesto como una alternativa quirúrgica para pacientes de sexo masculino con MAR alta y fístula recto-uretral $(6,7)$.

El DARAL ofrece ventajas sobre la anorectoplastia sagital posterior (ARPSP), como excelente visión de la fístula rectal, creación de un canal de descenso exacto para el intestino, y mínima invasión abdominal y herida perineal (6-9). Con el estimulador eléctrico la observación directa de la contracción del sling puborectal permite una evaluación funcional de la contractilidad y una precisión de el descenso colónico en el centro del complejo muscular (10).

EI DARAL y la ARPSP han descrito daño uretral durante el procedimiento; fístula recto-uretral recurrente o adquirida, divertículo uretral posterior, lesión uretral que lleva a estenosis o atresia uretral (2-7).

Existen pocos casos reportados de divertículo uretral tras la reparación de malformaciones anorectales, y todos ellos han sido manejados con extirpación quirúrgica $(2,4,11)$. Nosotros presentamos un caso de divertículo uretral tras DARAL con un resultado exitoso despues de un período de vigilancia activa.

\section{MATERIAL Y MÉTODO}

Se realiza el reporte de un recién nacido de término, de sexo masculino, que presentaba una malformación anorrectal (MAR) alta fue sometido a una colostomía el primer día de vida. Se controló antecedentes prenatales y embarazo, sin complicaciones obstétricas. El estudio de malformaciones asociadas mostró reflujo bilateral de bajo grado, un riñón en herradura y una hemi-vértebra torácica. No hubo signos de médula anclada. Se inició profilaxis antimicrobiana. A los 2 meses de edad un colograma distal diagnosticó una fístula recto-prostática. 


\section{RESULTADOS}

A los 3 meses de edad, fue sometido a un DARAL con un abordaje abdominal de 3 puertos. De antemano la fístula fue identificada bajo visión cistoscópica y se instalo un catéter uretral para facilitar el manejo posterior. Despues de una disección laparoscópica completa de el intestino distal, la fístula recto-prostática fue identificada, se extirpó el catéter uretral y se ligó la fístula con clips metálicos.

El centro del complejo esfinteriano fue identificado utilizando estimulación eléctrica perineal, y bajo guía laparoscópica un trocar de $10 \mathrm{~mm}$ fue insertado a través de él. El intestino distal fue recuperado con un grasper y el descenso anorrectal fue realizado sin tensión.

El manejo post operatorio fue con régimen cero. La ingesta oral se inició $48 \mathrm{hrs}$ luego de la cirugía, y un catéter vesical y antibióticos se mantuvo por 12 días. Fue dado de alta sin inconvenientes al 7ํ día de la cirugía.

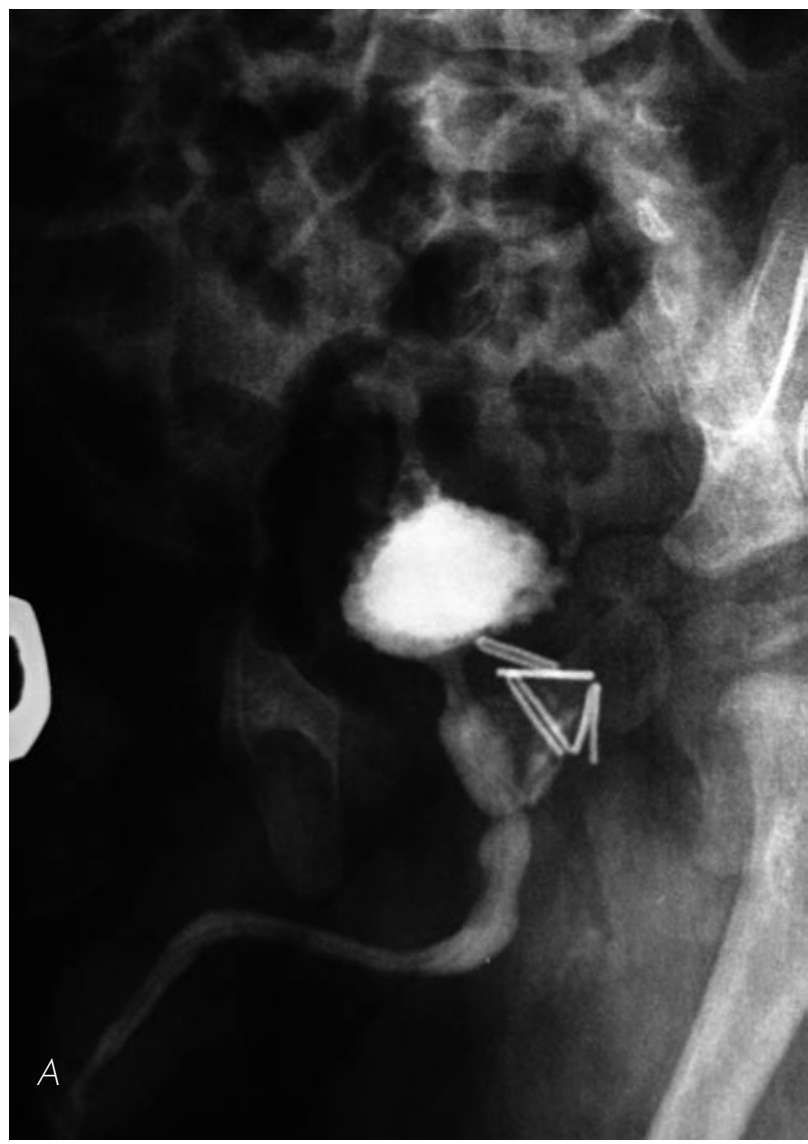

Despues de dos semanas se inició un programa de dilatación. Al 18으 día post operatorio, una cistografía miccional mostró una imagen diverticular de $15 \times 5 \mathrm{~mm}$ a nivel de la uretra membranosa (Figuras 1A y 1B), reflujo vesicoureteral grado 2 a derecha y grado 1 a izquierda. La ultrasonografía no mostraba dilatación. A los 6 meses no había problemas clínicos y una nueva cistorgrafía miccional mostró una uretra normal sin signos de divertículo o estenosis; persistencia del reflujo grado 2 a derecha y resolución del reflujo a izquierda (Figuras $2 \mathrm{~A}$ y2B).

Al año de edad la colostomía fue cerrada sin inconvenientes. Despues de 6 meses de seguimiento después de la última cirugía, se ha mantenido clínicamente bien, libre de ITU y con micción de flujo normal. No se ha presentado constipación o soiling en este periodo y él continúa con profilaxis antibiótica urinaria hasta una próxima evaluación de su reflujo vésico ureteral. Una última cistoscopía fue realizada a los 18 meses de edad la cual no mostró signos de divertículo.

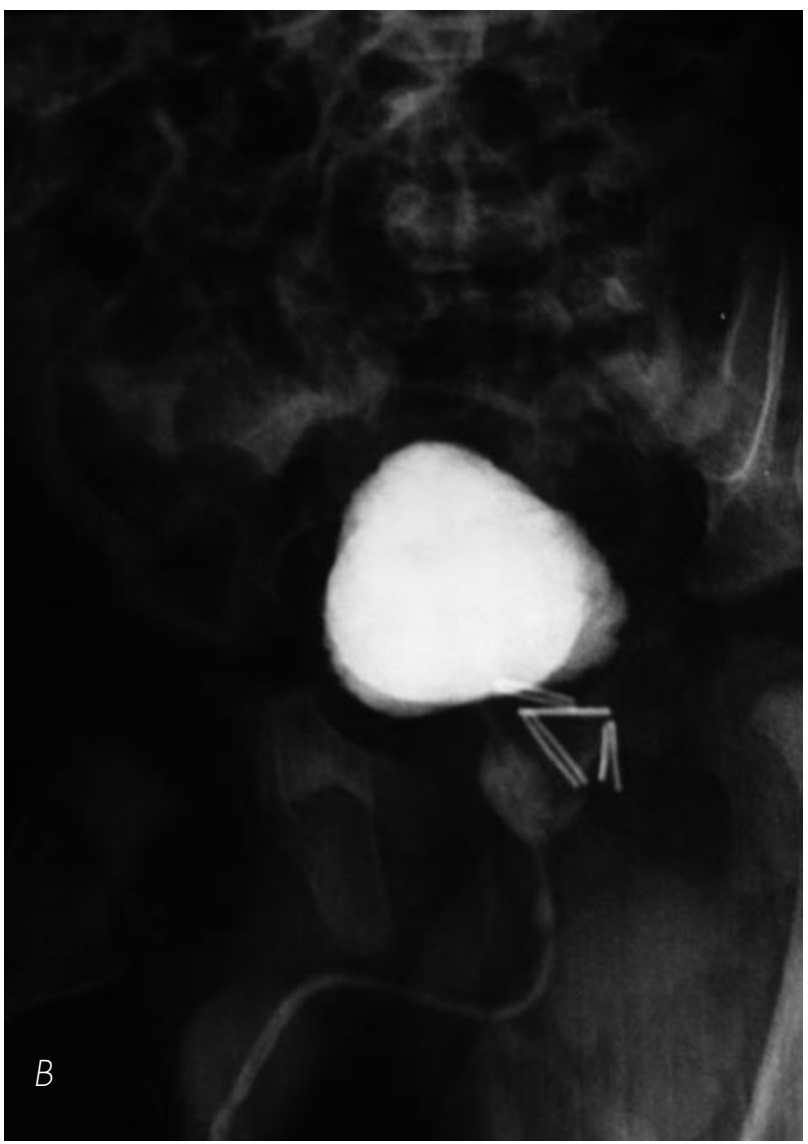

FIGURA IA y 1B. Cistografía miccional en el 18음 postoperatorio. Muestra una imagen diverticular de $15 x$ $5 \mathrm{~mm}$ a nivel de la uretra membranosa. 

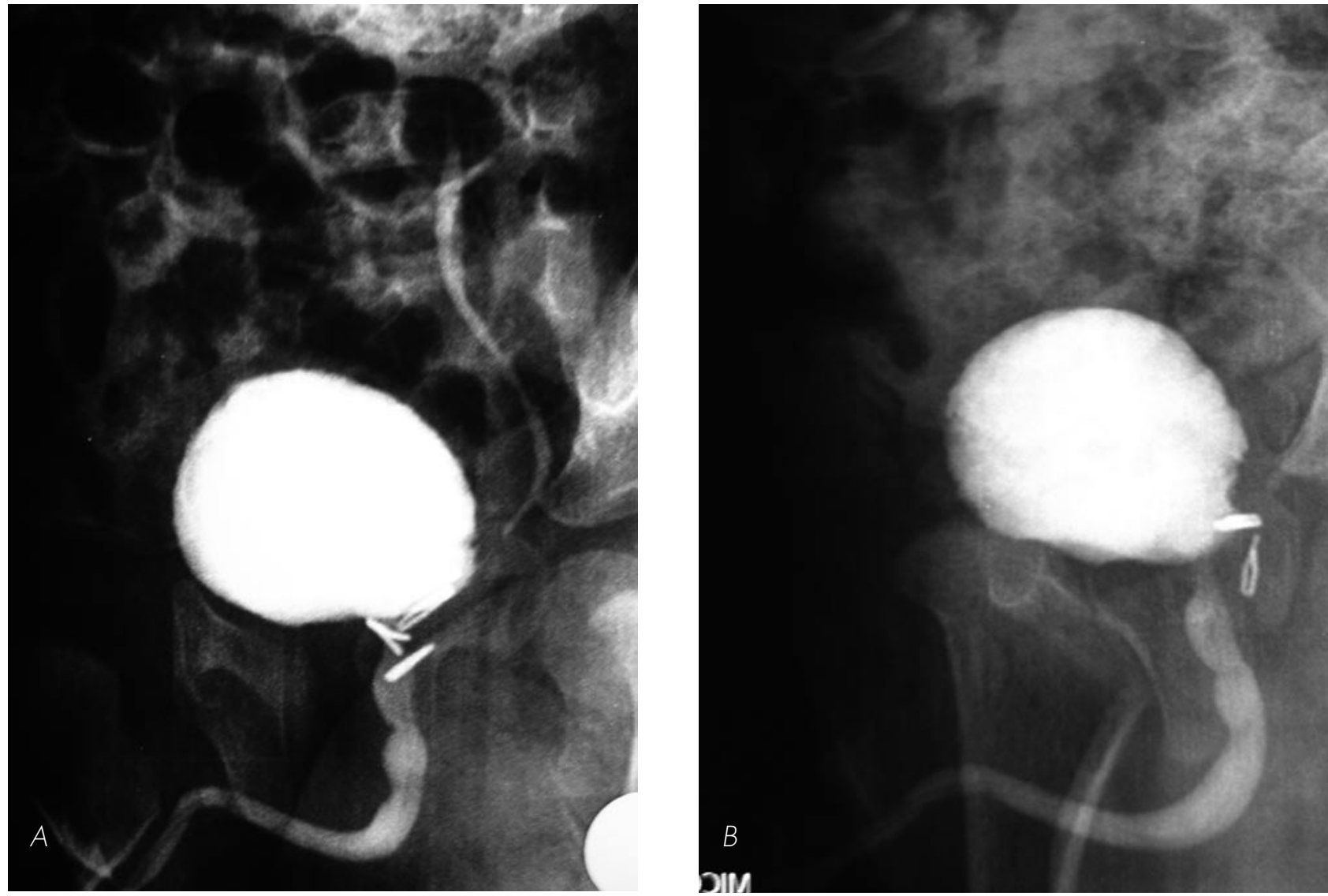

FIGURA 2A y 2B. Nueva cistografía miccional 6 meses después. Muestra una uretra normal sin signos de divertículo ni estenosis; persistencia del reflujo grado 2 a derecha y resolución del reflujo a izquierda.

\section{DISCUSIÓN}

Los divertículos uretrales son una rara patología, que en el varón podemos considerar como "primarios" de origen congénito y "secundarios" de origen adquirido (12). Los primarios corresponderían a ape-nas un $10 \%$ de todos los divertículos (12). El $90 \%$ del resto de divertículos reconocen origen adquirido: manipulaciones uretrales; compre-siones mantenidas con sistemas colec-tores o pinzas para incontinencias; complicaciones tras cirugías endoscópicas o uretroplastias e isquemias por decúbitos en los pacientes medulares (12).

En los niños, el divertículo uretral, puede ser visto despues de la reparación de MAR con una incidencia total de $2 \%$ de casos tras un abordaje abdominoperineal $(2,4)$. Pocos casos de divertículo uretral han sido descritos luego de DARAL (7). Los niños con esta complicación pueden presenter síntomas como alteración de la micción, incontinencia urinaria, infección urinaria, uropatía obstructiva, formación de cálculos (13) y transformación maligna $(2,3,14)$. Esa es la razón por lo que la extirpación quirúrgica del divertículo está siempre indicada.
El divertículo podría ser debido a un largo segmento de recto que quedó adherido a la uretra después del procedimiento (7) o debido a un proceso inflamatorio producido por la reacción a los clips. El tratamiento defendido para el divertículo uretral es la extirpación quirúrgica a través de una incisión sagital posterior (4) ○ por un abordaje abdominal (10).

Dificultades técnicas son más frecuentes en las fístulas recto-bulbares cuando se utiliza un DARAL (7), porque la fístula recto-bulbar es más baja que la fístula recto-prostática o recto-vesical y es más difícil de identificar en toda su extensión. Esta diferencia anatómica puede dejar un segmento distal de la fístula si no es disecada en forma completa, que puede persistir como un divertículo uretral. Tal como Koga et al propusieron nosotros realizamos una cistoscopia intra operatoria para identificar la fístula con un catéter que facilite la disección laparoscópica (7). Otras sugerencias son realizar una colonoscopia intra operatoria o usar un laparoscopio con ángulo de 30 ○ 40 grados. Esta técnica puede prevenir daños y complicaciones a las estructuras genitourinarias. 
Este es el primer caso de divertículo uretral luego de DARAL que ha sido manejado en forma conservadora. Reportes de casos anteriores, casi todos después de ARPSP, fueron tratados con extirpación quirúrgica, especialmente si eran sintomáticos $(11$, 15). Nosotros decidimos un abordaje observacional conservador porque el paciente estaba asintomático, la dilatación uretral no mostró un recto que permanezca unido a la uretra y no se encontró signos de obstrucción urinaria.

Los cirujanos pediatras deberían sospechar un divertículo uretral posterior en cualquier paciente con síntomas urinarios y una historia de reparación a través de un abordaje abdomino-perineal (2). Cuando un divertículo uretral evoluciona sin complicaciones, y no se encuentra una bolsa rectal remanente, éste se puede resolver eventualmente en forma espontánea. Vigilancia activa podría ser una opción en pacientes asintomáticos seleccionados, sin embargo se recomienda un mayor seguimiento para constituir una mejor evidencia que apoye esta medida.

\section{CONCLUSIÓN}

Este artículo sugiere que el DARAL es un abordaje factible para MAR, aunque el divertículo uretral es una de las principales preocupaciones. Puede evolucionar sin complicaciones, y eventualmente resolverse en forma espontánea. La vigilancia activa puede ser una opción en pacientes asintomáticos seleccionados, sin embargo se recomienda un mayor seguimiento para constituir una mejor evidencia que apoye esta medida.

\section{BIBLIOGRAFIAA y LECTURAS RECOMENDADAS (*lectura de interés $y^{* *}$ lectura fundamental)}

*1. Mickelson J, MacNeily A, Blair G. The posterior urethra in anorectal malformations. J Pediat Surg, 2007; 42, 585-7.

2. Hong A, Acuña M F, Peña A, Chaves L, Rodríguez G. Urologic Injuries Associated With Repair of Anorectal Malformations in Male Patients. J Pediatr Surg, 2002; 37, 339-44.

*3. Misra D, Chana J, Drake D P, Kiely E M, Spitz L. Operative trauma to the genitourinary tract in the treatment of anorectal malformations: 15 years' experience. Urol, 1996; 47: 559-62.

**4. Peña A, Grasshoff S, Levitt M. Reoperations in anorectal malformations J Pediat Surg, 2007; 42, 318-25.

5. Peña A, Hong A. Advances in the management of anorectal malformations. Am J Surg, 2000; 180:370-6.

**6. Georgeson KE, Inge TH, Albanese CT (2000) Laparoscopically assisted anorectal pull-through for high imperforate anus-a new technique. J Pediatr Surg 35: 927-31.

*7. Koga H, Okazaki T, Yamataka A, Kobayashi H, Yanai T, Lane G J, et al. Posterior urethral diverticulum after laparoscopic-assisted repair of hightype anorectal malformation in a male patient: surgical treatment and prevention Pediatr Surg Int, 2005; 21: 58.60.

8. Yamataka A, Segawa O, Yoshida R, Kobayashi $\mathrm{H}$, Kameoka S, Miyano T. Laparoscopic muscle electrostimulation during laparoscopy-assisted anorectal pull-through for high imperforated anus. J Pediatr Surg, 2001; 36:1659-61.

9. Yamataka A, Yoshida R, Kobayashi H, Lane GJ, Kurosaki Y, Segawa O, et al. Intraoperative endosonography enhances laparoscopy-assisted colon pull-through for high imperforate anus. J Pediatr Surg, 2003; 37:1657-60.

10. Lima M, Tursini S, Ruggeri G, Aquino A, Gargano T, De Biagi L, et al. Laparoscopically assisted anorectal pull-through for high imperforate anus: three years' experience. J Laparoendosc Adv Surg Tech A. 2006; 16(1):63-6.

11. Vinnicombe SJ, Good CD, Hall CM. Posterior urethral diverticula: a complication of surgery for high anorectal malformation. Pediatr Radiol, 1996; 26:120-6.

12. Ballesteros Sampol J., Cortadellas Angel R. y Juanpere Rodero N.: Divertículos adquiridos de uretra masculina. A propósito de siete casos. Revisión de la literatura. Arch Esp Urol, 2008; 61, 1: $1-6$.

13. Romero Selas E, Álvarez Castelo L, Ponce J, Sánchez J, Casas P, Janeiro J, et al. Divertículo de uretra habitado por litiasis. Arch Esp Urol, 2009; 62, 1: 449.

14. McLorie GA, Merguerian PA, DeMaria JE, et al. Injuries to the genitourinary system during a posterior sagittal approach and their repair. Br J Urol, 1998 (Suppl 2); 81:76.

**15. Peña A, Hong AR, Midulla P, Levitt M. Reoperative surgery for anorectal anomalies. Semin Pediatr Surg, 2003; 12:118-23. 